\title{
Mitochondria frozen with trehalose retain a number of biological functions and preserve outer membrane integrity
}

\author{
R Yamaguchi ${ }^{1}$, A Andreyev ${ }^{2}$, AN Murphy ${ }^{3}$, GA Perkins ${ }^{4}$, MH Ellisman $^{4}$ and DD Newmeyer ${ }^{1, \text { * }}$
}

In apoptosis, Bcl-2-family proteins regulate the barrier function of the mitochondrial outer membrane (MOM), controlling the release of proapoptotic proteins from the intermembrane space into the cytoplasm. This process can be studied in vitro with freshly isolated mouse liver mitochondria. Unfortunately, mitochondria frozen/thawed in standard sucrose-mannitol buffers become leaky and useless for apoptosis research. However, here we show that mitochondria frozen in buffer containing the sugar, trehalose, maintained MOM integrity and responsiveness to Bcl-2-family proteins, much like fresh mitochondria. Trehalose also preserved ultrastructure, as well as biological functions such as ATP synthesis, calcium-induced swelling, transmembrane potential, and the import and processing of protein precursors. However, bioenergetic function was somewhat reduced. Thus, trehalose-frozen mitochondria retained most of the biological features of mitochondria including MOM integrity. Although not ideal for studies involving bioenergetics, this method will facilitate research on apoptosis and other mitochondrial functions that rely on an intact MOM.

Cell Death and Differentiation (2007) 14, 616-624. doi: doi:10.1038/sj.cdd.4402035; published online 15 September 2006

Since the 1970s, there have been a number of attempts to freeze isolated mitochondria for storage, with various degrees of success. In most of these studies, the functionality of frozen-thawed mitochondria was assessed with regard to bioenergetic parameters. In a recent and relatively successful attempt, frozen mitochondria were reported to have roughly $50 \%$ of normal respiratory function, and the mitochondria were well coupled. ${ }^{1}$ However, in all these studies, partial retention of respiratory functions was accomplished at the expense of disrupting mitochondrial outer membrane (MOM) integrity.

In recent years, it became apparent that mitochondria play another important role in the cell besides respiration: that of regulating apoptosis. Permeabilization of the MOM is a critical step in the mitochondrial apoptotic pathway, ${ }^{2-5}$ leading to the release of soluble proteins such as cytochrome $c$, Smac and Omi from the mitochondrial intermembrane space (IMS). Once these proapoptotic proteins are released into cytosol, the Apaf-1 apoptosome and caspases are activated, resulting in death of the cell. MOM permeabilization is controlled primarily by the Bcl-2 family of proteins. In particular, the Bak and Bax proteins, when activated, form pores in the MOM, allowing IMS proteins to be released.

Much of what we know about the mitochondrial apoptotic pathway has been determined from experiments using isolated mouse liver mitochondria (which contain primarily
Bak, not Bax). The outer membranes of these isolated organelles tend to deteriorate over time and rupture immediately upon freeze-thaw. Thus, the functional analysis of mouse liver mitochondria (and presumably mammalian mitochondria from other sources) currently requires freshly prepared organelles. To reduce the need for costly and timeconsuming preparations, we sought to identify a storage medium that preserves mitochondrial function, particularly of the outer membrane. To that end, we analyzed the integrity of MOMs after rapid freeze-thaw in buffers containing various carbohydrates. Mitochondria frozen in almost all these solutions rapidly lost their MOM integrity and released cytochrome $c$ when thawed and incubated at $37^{\circ} \mathrm{C}$. However, we found that freezing mitochondria in buffer containing one particular sugar, trehalose, resulted in the preservation of MOM barrier function.

Trehalose is a naturally occurring disaccharide composed of two glucose molecules connected by an alpha, alpha-1,1 linkage. This sugar is implicated in the ability of plants and animals to withstand prolonged periods of desiccation, a process called xerobiosis. It has been shown that trehalose can protect proteins and cellular membranes from harmful effects of dehydration. ${ }^{6}$ Womersley and colleagues ${ }^{7}$ demonstrated that trehalose was more effective in preserving the structural and functional integrity of $\mathrm{Ca}^{2+}$-transporting microsomes under dehydrating conditions than other

\footnotetext{
${ }^{1}$ Department of Molecular Immunology, La Jolla Institute for Allergy and Immunology, La Jolla, CA, USA; ${ }^{2}$ Department of Chemistry and Biochemistry, University of California San Diego, La Jolla, CA, USA; ${ }^{3}$ Department of Pharmacology, University of California San Diego, La Jolla, CA, USA and ${ }^{4}$ Department of Neurosciences and National Center for Microscopy and Imaging Research, University of California San Diego, La Jolla, CA, USA

*Corresponding author: D Newmeyer, Department of Molecular Immunology, La Jolla Institute for Allergy and Immunology, 9420 Athena Circle, La Jolla, CA 92037, USA. Tel: + 1-858-752-6658; Fax: + 1-206-333-0179; E-mail: don@liai.org

Keywords: mitochondria, apoptosis, freezing, trehalose, outer membrane, cryopreservation

Abbreviations: MOM, mitochondrial outer membrane; IMS, intermembrane space; TMRE, tetramethylrhodamine methyl ester; CCCP, carbonyl cyanide m-chlorophenylhydrazone; G3P, glycerol-3-phosphate; SDS-PAGE, sodium dodecyl sulfate-polyacrylamide gel electrophoresis; N/C-Bid, recombinant cleaved Bid; PTP, permeability transition pore; Su9-DHFR, fusion of the mitochondrial targeting signal of Su9 with dihydrofolate reductase

Received 13.12.05; revised 28.7.06; accepted 28.7.06; Edited by JC Martinou; published online 15.9.06
} 
carbohydrates, including lactose, maltose, cellobiose, sucrose, glucose, fructose, sorbitol, raffinose, myo-inositol, glycerol and alcohol sugars. In another study, trehalose was also found to be more efficient than glycerol and dimethylsulfoxide in the stabilization of sarcoplasmic reticulum during freeze-thaw. ${ }^{8}$

Here we provide a method for the long-term storage of frozen mouse liver mitochondria in trehalose-containing buffer and show that trehalose-frozen mitochondria retain a number of their important biological functions, most notably the preservation of MOM integrity.

\section{Results}

Cytochrome $c$ is retained in trehalose-frozen mitochondria. To identify a suitable storage buffer that keeps mitochondria intact after freeze-thaw, we isolated mitochondria in buffers containing various concentrations of sugar and analyzed cytochrome $c$ retention over time. We tested the following solutions: $0.6 \mathrm{mM}$ sorbitol, $250-300 \mathrm{mM}$ sucrose, 250-300 mM mannitol, $200 \mathrm{mM}$ sucrose $/ 75 \mathrm{mM}$ trehalose, $200 \mathrm{mM}$ mannitol $/ 75 \mathrm{mM}$ trehalose, $200 \mathrm{mM}$ trehalose $/ 75 \mathrm{mM}$ sucrose, $200 \mathrm{mM}$ trehalose $/ 75 \mathrm{mM}$ mannitol or 250-300 mM trehalose, all containing $N$-2-hydroxyl piperazine- $N$-2-ehane sulfonic acid (HEPES)-KEE mix (10 mM HEPES-KOH pH 7.7, $10 \mathrm{mM} \mathrm{KCl,} 0.1 \%$ bovine serum albumin (BSA), $1 \mathrm{mM}$ ethylene diaminetetra acetic acid (EDTA) and $1 \mathrm{mM}$ ethylene glycol-bis ( $b$-aminoethyl ether) (EGTA)). We found that in almost all cases, if mitochondria were not frozen, they retained cytochrome $c$ for $3-4 \mathrm{~h}$ at $22^{\circ} \mathrm{C}$ (data not shown). However, mitochondria frozen in almost all of these buffers lost a portion of their cytochrome $c$ within the first $15 \mathrm{~min}$ after thawing at $22^{\circ} \mathrm{C}$, suggesting that the freeze-thaw process damages the MOM (Figure 1a). Of the solutions tested, only $300 \mathrm{mM}$ trehalose was effective for preserving cytochrome $c$ content: mitochondria frozen and thawed in $300 \mathrm{mM}$ trehalose buffer retained almost all their cytochrome $c$ for at least $45 \mathrm{~min}$ (Figure 1a and b).

Similar results were obtained at the more physiologically relevant temperature of $37^{\circ} \mathrm{C}$. When mitochondria that had been frozen in sucrose-mannitol buffer were incubated at $37^{\circ} \mathrm{C}, 95 \%$ of their cytochrome $c$ was lost during the first $15 \mathrm{~min}$. Incidentally, the same mitochondria lost only $20-30 \%$ of their content of cytochrome $c$ when incubated at $22^{\circ} \mathrm{C}$ (Figure 1b, compare left middle and left bottom panels). The reason for this temperature dependence of cytochrome $c$ efflux from mitochondria frozen/thawed in sucrose-mannitol buffer is unknown, but is hypothesized to involve temperaturedependent changes in the organization of cristae. ${ }^{9}$

In contrast, trehalose-frozen mitochondria retained almost all cytochrome $c$ for at least $45 \mathrm{~min}$ either at 22 or $37^{\circ} \mathrm{C}$ (Figure 1b, right panels). Further analysis demonstrated that trehalose-frozen mitochondria retained cytochrome $c$ for at least $2-3 \mathrm{~h}$ at $37^{\circ} \mathrm{C}$ (data not shown). Over $95 \%$ of total cytochrome $c$ was retained in trehalose-frozen mitochondria during a $45-\mathrm{min}$ incubation at $37^{\circ} \mathrm{C}$ (Figure 1C), compared to only $7 \%$ for mitochondria frozen in sucrose-mannitol buffer (data not shown). We conclude that trehalose-frozen a

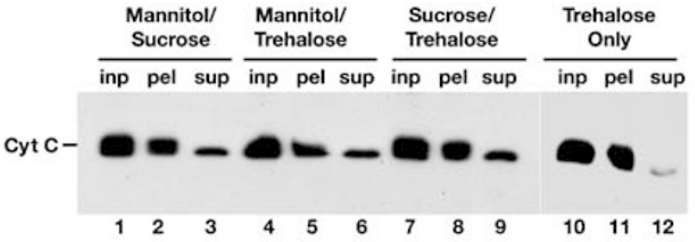

b
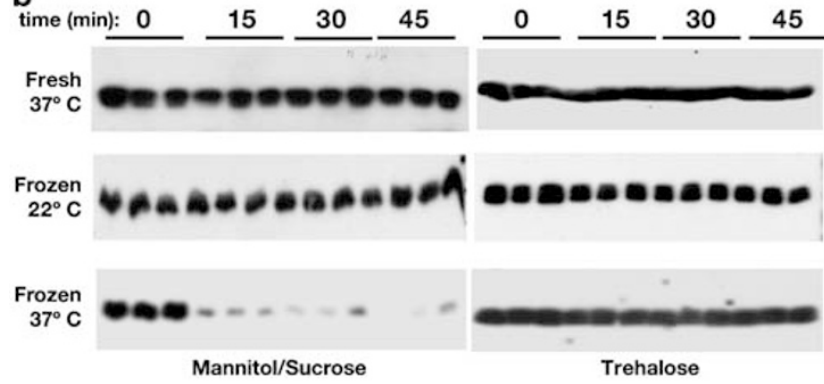

C

$\%$ of mitochondrial input

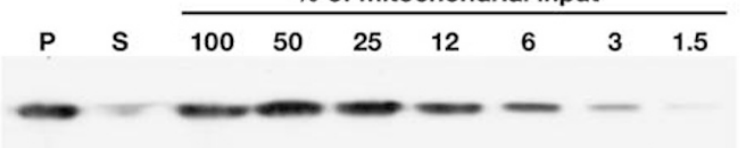

Figure 1 Retention of cytochrome $c$ in trehalose-frozen mitochondria. (a) Mouse mitochondria were frozen in buffers containing each of the following sugar mixtures: $67 \mathrm{mM}$ sucrose and $200 \mathrm{mM}$ mannitol (lanes 1-3), $67 \mathrm{mM}$ mannitol and $200 \mathrm{mM}$ trehalose (lanes 4-6), $67 \mathrm{mM}$ sucrose and $200 \mathrm{mM}$ trehalose (lanes 7-9), or $300 \mathrm{mM}$ trehalose (lanes 10-12) and quickly thawed in the same buffer containing $80 \mathrm{mM} \mathrm{KCl}$. Aliquots of thawed mitochondria were either suspended in SDS sample buffer (lanes 1, 4, 7 and 10) or incubated at $22^{\circ} \mathrm{C}$ for $5 \mathrm{~min}$ and centrifuged for $10 \mathrm{~min}$ to separate mitochondrial pellets (lanes 2, 5, 8 and 10) from supernatants (lanes 3, 6, 9 and 12). Samples were resolved by SDS-15\% PAGE and analyzed by Western blot using anti-cytochrome $c$ antibody. (b) Fresh or frozen mitochondria in either the sucrose/mannitol (left) or trehalose (right) buffer were resuspended in the same buffer and incubated either at 22 or $37^{\circ} \mathrm{C}$ for $0-45 \mathrm{~min}$. Mitochondria were isolated by centrifugation, triplicates of samples were subjected to SDS-PAGE, and the cytochrome $c$ content of the samples analyzed by Western blotting. (c) Trehalose-frozen mitochondria were resuspended in trehalose buffer and incubated at $37^{\circ} \mathrm{C}$ for $45 \mathrm{~min}$. The sample was fractionated by centrifugation and immunoblotted along with a series of dilutions of unfractionated samples, to estimate the percentage of released cytochrome $c$. The pellet fraction $(P)$ contained more than $95 \%$ and the supernatant (S) less than $5 \%$ of the cytochrome $c$ input. Although the rate of spontaneous release varied between mitochondrial preparations, it was usually below $5 \%$ of the total mitochondrial cytochrome $c$ in the input

mitochondria retain cytochrome $c$ at $37^{\circ} \mathrm{C}$ much more effectively than mitochondria frozen in the standard buffer.

Trehalose-frozen mitochondria release cytochrome $c$ in response to recombinant cleaved Bid. The retention of cytochrome $c$ in trehalose-frozen mitochondria suggested that their OMs were intact. Alternatively, it was possible that the process of freezing mitochondria in the presence of trehalose altered the morphology of the mitochondria in some way, resulting in cytochrome $c$ being trapped in compartments other than the IMS, such as the matrix. Therefore, we examined the ability of trehalose-frozen mitochondria to release cytochrome $c$ in response to $\mathrm{BH} 3-$ only proteins of proapoptotic Bcl-2 family members that activate Bax and Bak. 
We compared the response of freshly isolated and trehalose-frozen mitochondria to $\mathrm{BH} 3-$ only proteins first by incubating the organelles with $10 \mathrm{nM}$ recombinant cleaved Bid (N/C-Bid) at $37^{\circ} \mathrm{C}$. The results showed that both fresh and trehalose-frozen mitochondria released almost all of their cytochrome $c$ within $15 \mathrm{~min}$ (Figure 2a). Thus, trehalosefrozen mitochondria released cytochrome $c$ in response to cleaved Bid with kinetics similar to those of freshly isolated mitochondria.

$\mathrm{N} / \mathrm{C}$-Bid also induced the release of Omi, another IMS protein, from trehalose-frozen mitochondria. When trehalosefrozen mitochondria were treated with $\mathrm{N} / \mathrm{C}-\mathrm{Bid}$ and incubated at $37^{\circ} \mathrm{C}$, Omi and cytochrome $c$ began to appear in the supernatants at $12 \mathrm{~min}$, and by $24 \mathrm{~min}$ both Omi and cytochrome $c$ were almost entirely in the supernatants (Figure 2b, middle two panels). On the other hand, VDAC, a voltage-dependent channel protein that is integral to the MOM, was retained in the mitochondrial pellets throughout the experiment, as expected (Figure $2 \mathrm{~b}$, bottom two panels).

To test the specificity of the response to N/C-Bid in these experiments, we added a 10-fold excess of the antiapoptotic Bcl-2-family protein, Bcl- $x_{L}$, known to bind to and inhibit $\mathrm{N} / \mathrm{C}$-Bid. Bcl- $\mathrm{x}_{\mathrm{L}}$ blocked the release of cytochrome $c$ and Omi, suggesting that the regulation of MOM permeabilization by Bcl-2-family proteins ${ }^{10}$ was normal in these frozen-thawed
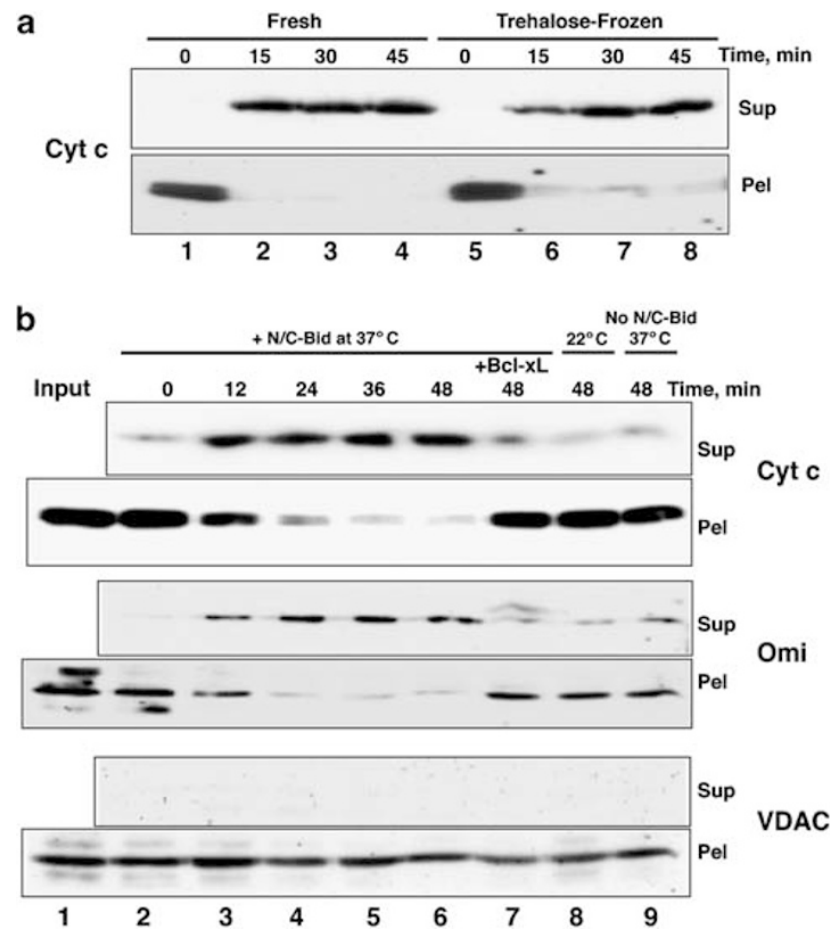

Figure 2 Trehalose-frozen mitochondria retained MOM integrity. (a) Fresh and trehalose-frozen mitochondria were resuspended in the assay buffer and incubated with $10 \mathrm{nM}$ of $\mathrm{N} / \mathrm{C}$-Bid at $37^{\circ} \mathrm{C}$ for $0,15,30$ or $45 \mathrm{~min}$. Mitochondrial pellets were precipitated by centrifugation and pellets and the cytochrome $c$ content of the samples was analyzed by Western blotting. (b) Trehalose-frozen mitochondria were resuspended in the assay buffer supplemented with either $10 \mathrm{nM} \mathrm{N} / \mathrm{C}$-Bid (lanes 2-

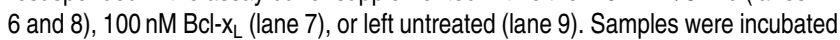
at $37^{\circ} \mathrm{C}$ (lanes $2-7$ and 9 ) or at $22^{\circ} \mathrm{C}$ (lane 8). At the indicated time points, samples were centrifuged to separate pellets from supernatants and their contents analyzed by Western blot mitochondria. We also observed that N/C-Bid-induced release of cytochrome $c$ and Omi only occurred at physiological temperature $\left(37^{\circ} \mathrm{C}\right)$. When mitochondria were incubated with the same concentration of $\mathrm{N} / \mathrm{C}-\mathrm{Bid}$ at $22^{\circ} \mathrm{C}$ for as long as $45 \mathrm{~min}$, neither cytochrome $c$ nor Omi was released (lane 8). Similar results were obtained using peptides corresponding to the $\mathrm{BH} 3$ domains of $\mathrm{Bid}$ and $\left.\mathrm{Bim}^{11}\right)$ : these peptides $(20 \mu \mathrm{M})$ triggered the release of cytochrome $c$ and Omi from trehalosefrozen mitochondria with kinetics comparable to those seen with freshly isolated mitochondria (data not shown).

In summary, trehalose-frozen mitochondria responded to BH3-only proteins by releasing cytochrome $c$ and Omi from their IMS. The evidence strongly suggests that MOMs of trehalose-frozen mitochondria remained intact after freezethaw and also remained responsive to $\mathrm{BH}$-only proteins. We also observed that N/C-Bid-induced oligomerization of Bak in trehalose-frozen mitochondria occurred in the same manner as with fresh mitochondria (not shown), a further indication that the MOMs of trehalose-frozen mitochondria were intact and functional.

ATP synthesis in trehalose-frozen mitochondria. To ensure further that trehalose-frozen mitochondria are indeed biologically similar to freshly prepared mitochondria, we assayed other biological properties. ATP synthesis is a critical function of mitochondria. We first compared the ATP content of 2-week-old trehalose-frozen mitochondria with that of freshly isolated mitochondria, using bioluminescence assays (Figure 3, 'input'). Frozen-thawed mitochondria contained less than half the amount of ATP ( $\sim 19 \mathrm{ng}$ of $\mathrm{ATP} / \mu \mathrm{g}$ protein) present in fresh mitochondria ( $\sim 40 \mathrm{ng}$ of $\mathrm{ATP} / \mu \mathrm{g}$ protein). This difference suggests that a slow hydrolysis of ATP occurred during storage.

To stimulate ATP synthesis, mitochondria were suspended in buffer containing $67 \mu \mathrm{M}$ ADP and incubated at $37^{\circ} \mathrm{C}$. At the outset, the ATP concentration of fresh mitochondrial suspensions (10 $\mu \mathrm{g}$ total protein) was about $95 \mu \mathrm{M}$, and within $30 \mathrm{~min}$, it increased by $20 \%$ to $118 \mu \mathrm{M}$ (Figure 3, top). No further increase was seen with longer incubation (data not shown). The addition of glycerol-3-phosphate (G3P) did not stimulate ATP synthesis more than ADP alone and indeed produced a slightly smaller increase, to $115 \mu \mathrm{M}$. With suspensions of frozen mitochondria, in contrast, $67 \mu \mathrm{M}$ ADP stimulated a $110 \%$ increase in ATP concentration in $30 \mathrm{~min}$, producing a final ATP concentration of $95 \mu \mathrm{M}$, comparable to that of unstimulated fresh mitochondria (Figure 3, bottom). In the presence of both ADP and G3P, the ATP concentration of frozen mitochondria increased by $155 \%$, reaching nearly $115 \mu \mathrm{M}$. Again, this concentration is comparable to those of $\mathrm{ADP}$ or ADP + G3P-stimulated fresh mitochondria.

Antimycin $A$ is a potent inhibitor of electron transfer through complex III. When antimycin A was added to either ADPstimulated or ADP + G3P-stimulated frozen mitochondria (Figure 3), no increases in ATP levels were seen upon incubation; rather, the ATP concentrations declined to less than $20 \mu \mathrm{M}$ in both cases. When fresh mitochondria were treated with antimycin A along with ADP or ADP + G3P, there were precipitous declines in ATP concentration to nearly $15 \mu \mathrm{M}$. The larger decreases seen in fresh mitochondria in the presence of antimycin A may indicate faster rates of ATP 


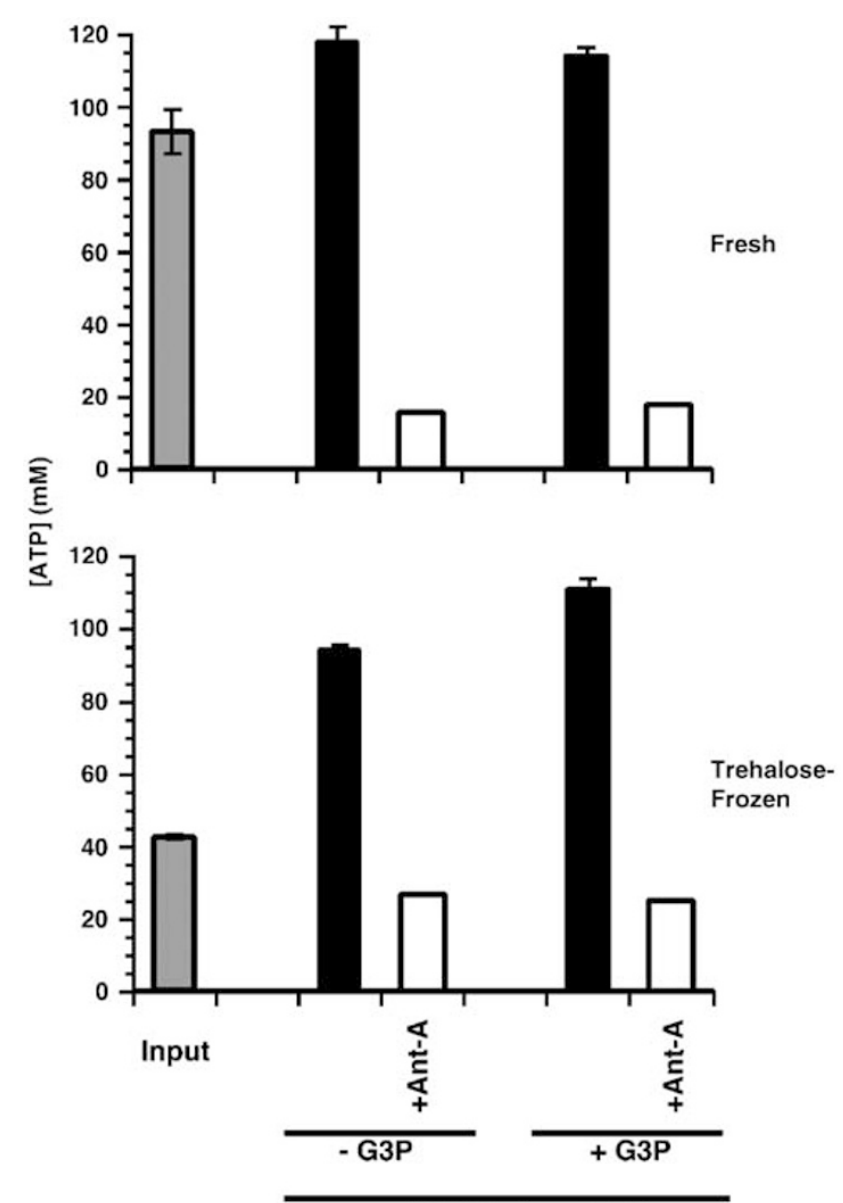

$+\mathrm{ADP}, 30 \mathrm{~min}$ at $37^{\circ} \mathrm{C}$

Figure 3 Trehalose-frozen mitochondria can synthesize ATP. Fresh and trehalose-frozen mitochondria were incubated in ATP synthesis assay buffer and incubated at $37^{\circ} \mathrm{C}$ for $30 \mathrm{~min}$. To stimulate ATP synthesis, $67 \mu \mathrm{M}$ of ADP was added. Where indicated, $5 \mathrm{mM} \mathrm{G3P}, 0.2 \mu \mathrm{M}$ antimycin $\mathrm{A}$ (Ant-A) or both were added to samples. All samples were analyzed in triplicates and ATP concentrations were plotted. The experiment shown was representative of three independent experiments

hydrolysis in freshly prepared mitochondria. These data suggest firstly that the increases in ATP concentration we observed with the addition of ADP and ADP + G3P were mitochondrially generated and secondly that there is constitutive ATP hydrolysis in the mitochondria. Taken together, our data clearly indicate that trehalose-frozen mitochondria are capable of ATP synthesis.

Trehalose-frozen mitochondria swell in response to increased calcium concentration. Cytosolic calcium concentrations are tightly regulated by the endoplasmic reticulum and mitochondria. ${ }^{12-14}$ Calcium is imported into, and exported out of, the mitochondrial matrix through ion channels located in the inner membrane. Calcium sequestered in mitochondria plays important physiological roles including: (1) controlling the rate of oxidative phosphorylation, (2) modifying the shape of cytosolic $\mathrm{Ca}^{2+}$ pulses or transients and (3) under conditions of $\mathrm{Ca}^{2+}$ overload, inducing the permeability transition (PT), which a
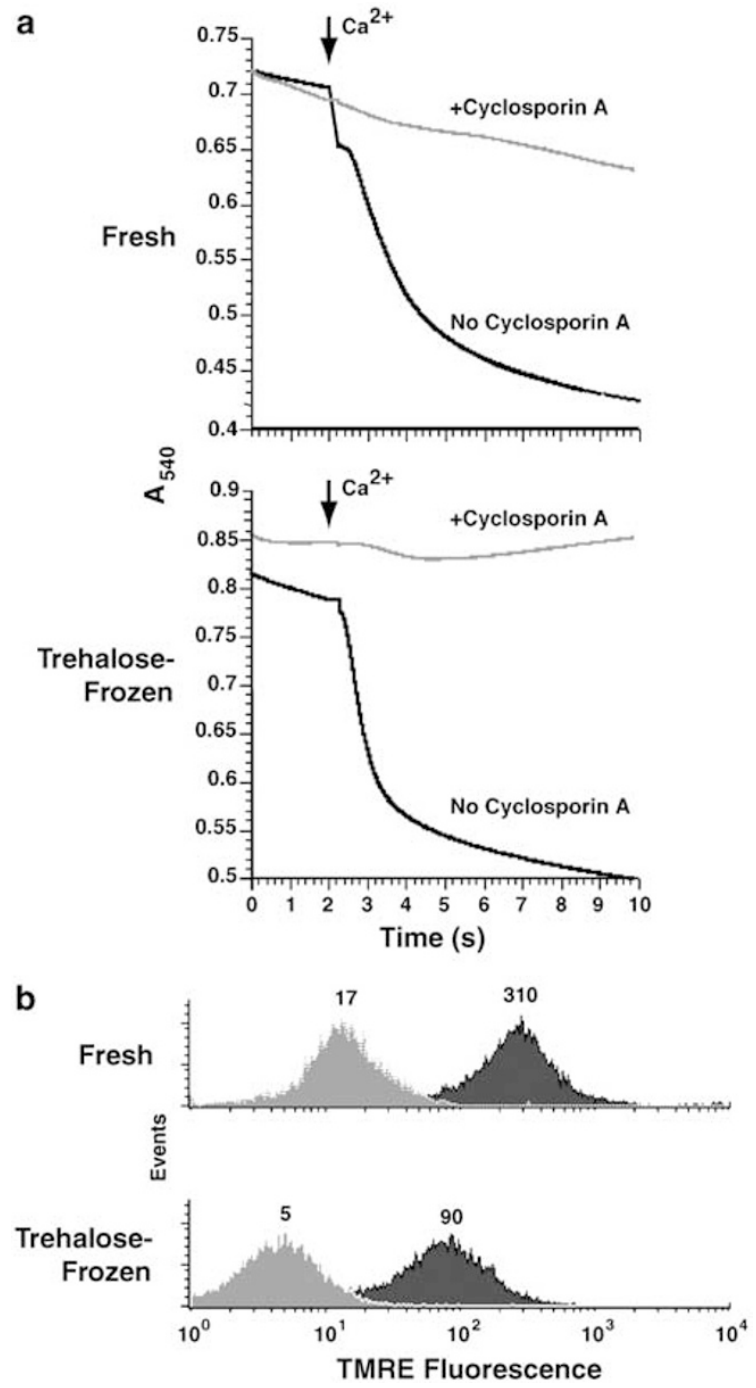

Figure 4 Trehalose-frozen mitochondria display normal PT. (a) Fresh and frozen mitochondria were resuspended in isotonic buffer (see Materials and Methods) with or without Cyclosporin A and light scattering was measured 5 times every second at $540 \mathrm{nM}$ for $10 \mathrm{~min}$. For each fresh or frozen mitochondria sample, samples containing $25 \mu \mathrm{M}$ Cyclosporin A were analyzed simultaneously. At 2 min, $100 \mu \mathrm{M}$ calcium was added to both samples and measurement resumed for another $8 \mathrm{~min}$. In both frozen and fresh mitochondria, with the addition of calcium, absorbance at $540 \mathrm{~nm}$ changes dramatically in the absence of cyclosporin A. (b) Mitochondria were suspended in PBS and $100 \mathrm{nM}$ TMRE was added to the suspensions which were mixed well and immediately analyzed by flow cytometry (black fill). CCCP $(1 \mu \mathrm{M})$ was added to the samples to dissipate transmembrane potential (gray fill)

causes mitochondrial matrix swelling, rupture of the MOM and the release of cytochrome $c$, followed by cell death.

Thus, we determined whether trehalose-frozen mitochondria can respond to increasing concentrations of calcium by activating the permeability transition pore (PTP). Calciuminduced mitochondrial swelling was assayed by measuring the decrease in light scattering at $540 \mathrm{~nm}$. The addition of exogenous calcium $(100 \mu \mathrm{M}$, or $\sim 200 \mathrm{nmol} / \mathrm{mg}$ of mitochondrial protein) induced a comparable degree of swelling in fresh and trehalose-frozen mitochondria (Figure 4a). Pretreatment with $25 \mu \mathrm{M}$ cyclosporin A, an inhibitor of PTP, blocked the 
swelling in both cases. This suggests that in both cases, swelling was caused by calcium-induced PTP opening, as expected.

\section{Trehalose-frozen mitochondria retain transmembrane} potential. Mitochondrial electron transport generates an electrochemical proton gradient of approximately 180$200 \mathrm{mV}$ across the inner membrane that drives ATP synthesis. As trehalose-frozen mitochondria are capable of ATP synthesis, we expected the transmembrane potential, $\Delta \Psi_{\mathrm{m}}$, to be present in frozen mitochondria. We used flow cytometry to compare the retention of the potentiometric fluorescent dye tetramethylrhodamine methyl ester (TMRE) in fresh vs trehalose-frozen mitochondria. Both types of mitochondria retained TMRE (Figure 4b; graphs filled in black), and the amount of retained TMRE was greatly reduced by the uncoupler, carbonyl cyanide $m$ chlorophenylhydrazone (CCCP) (Figure 4b; graphs filled in gray), indicating that both mitochondrial preparations possessed a measurable inner membrane potential. In this experiment, we did observe a lower overall TMRE fluorescence in the frozen mitochondria. This may merely reflect variation in staining perhaps due to different total numbers of mitochondria in the two preparations, or it may reflect altered respiratory function in the trehalose-frozen mitochondria (see below). Addition of a higher concentration of CCCP $(10 \mu \mathrm{M})$ did not cause further loss of TMRE staining (data not shown) suggesting that CCCP had dissipated $\Delta \Psi_{\mathrm{m}}$ completely in each case.

Trehalose-frozen mitochondria are capable of protein import and processing. Most mitochondrial proteins are encoded on nuclear genes and synthesized as precursor proteins in the cytoplasm. These precursors are imported into the organelle via a multistep pathway that includes binding to surface receptors on the MOM, unfolding of the precursor, translocation across both mitochondrial membranes, processing to the mature form, refolding of the imported protein and assembly into multisubunit enzyme complexes.

To investigate whether trehalose-frozen mitochondria are capable of protein import, we introduced $a{ }^{35} \mathrm{~S}$-labeled fusion of the mitochondrial targeting signal of Su9 with dihydrofolate reductase (Su9-DHFR). ${ }^{15}$ As a negative control, we used the nuclear export receptor Crm1. When trehalose-frozen mitochondria were incubated at $37^{\circ} \mathrm{C}$ with an energy regenerating system in AT buffer, Su9-DHFR was observed in the mitochondrial pellets within 30-60 min (Figure 5, lanes 4, 6 and 8). The mature form of Su9-DHFR, indicated by mSu9 in Figure 5, appears in the pellet fractions at 30-90 min, indicating that Su9-DHFR maturation took place in the mitochondria. As expected, very little Crm1 was associated with mitochondrial pellets as it remains in the cytosol (lanes 2-9). When we added a metalloprotease inhibitor, phenanthroline, Su9-DHFR was still associated with the mitochondria. However, the absence of the mature form of Su9-DHFR in the mitochondrial pellet indicated that although the protein was imported into mitochondria, its processing was blocked by phenanthroline (lanes 16 and 17), suggesting that processing of Su9-DHFR is dependent on metalloprotease activities.

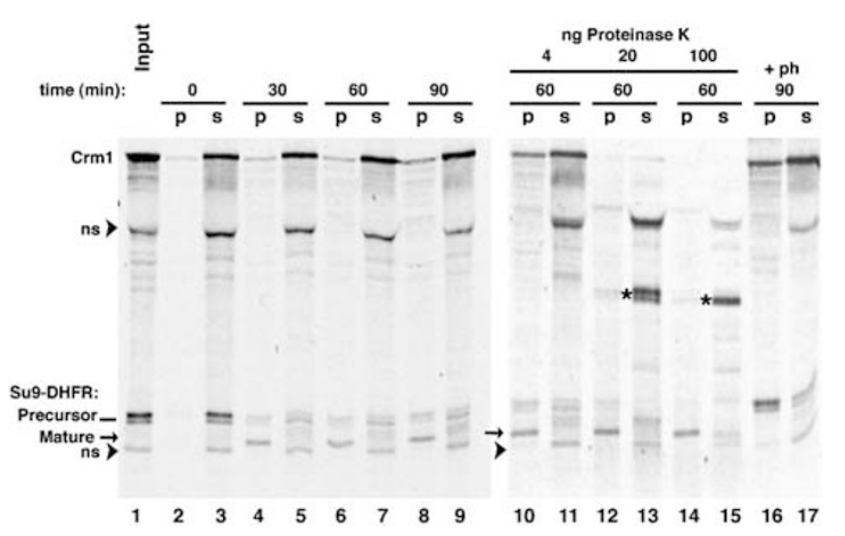

Figure 5 Trehalose-frozen mitochondria can import a model precursor protein and display mitochondrial peptidase activity. Frozen mitochondria were resuspended in AT buffer containing $80 \mathrm{mM} \mathrm{KCl}$ and supplemented with an energy regenerating system and ${ }^{35} \mathrm{~S}$-labeled Su9DHFR and $\mathrm{Crm} 1$ proteins made in rabbit reticulocyte lysates. At the time points indicated at the top, mitochondria were pelleted and supernatants $(s)$ and pellets $(p)$ were analyzed by SDS-PAGE and phosphorimaging. Following the import reaction, the samples analyzed in lanes 1015 were treated with $4-100 \mathrm{ng} / \mathrm{ml}$ of proteinase K for $30 \mathrm{~min}$. Samples analyzed in lanes 16 and 17 contained $200 \mu \mathrm{M}$ phenanthroline to inhibit metalloproteinase activities in the mitochondria. Positions of Su9-DHFR precursor and mature processed forms (arrows) are indicated. Arrowheads indicate nonspecific proteins labeled in rabbit reticulocyte lysates (ns). The asterisks $\left(^{*}\right)$ in lanes 13 and 14 indicate degradation products of Crm1

We used proteinase $\mathrm{K}$ digestion as an assay for protein import. Limited concentrations of proteinase $\mathrm{K}$ can degrade proteins outside mitochondria, whereas proteins inside the MOM or imported into the matrix are largely resistant to degradation. We added three different concentrations of proteinase $\mathrm{K}$ to mitochondria that had been incubated with Su9-DHFR for $60 \mathrm{~min}$. After a further $30 \mathrm{~min}$ incubation, we observed the disappearance of Su9-DHFR from the supernatant. Not surprisingly, the reduction of protein in the supernatant was greater with higher amounts of proteinase $\mathrm{K}$ added. Crm1 degradation in the supernatant was also observed with addition of proteinase $\mathrm{K}$ (asterisks in lanes 1015 denote apparent degradation products). We also note that at higher concentrations of proteinase $\mathrm{K}$, only the mature form of Su9-DHFR remained in the mitochondrial pellet (lane 14). Thus our data indicate that the mature form of Su9-DHFR had translocated into mitochondria and was inaccessible to proteinase $\mathrm{K}$. We also tested the mitochondrial import of natural substrates such as Mgm1/OPA1, Dpk1, AIF1 and others. Trehalose-frozen mitochondria efficiently imported and processed each of these (data not shown).

Trehalose preserves ultrastructure of mitochondria. To examine the degree of structural preservation that accompanied the freezing processes, we used transmission electron microscopy. We observed significant differences between isolated mitochondria frozen in trehalose or mannitol/sucrose revealed (Figure 6). Isolated mitochondria often display a range of conformational states from 'orthodox' to 'condensed' with the majority being in the condensed state (for definitions of orthodox and condensed, see 

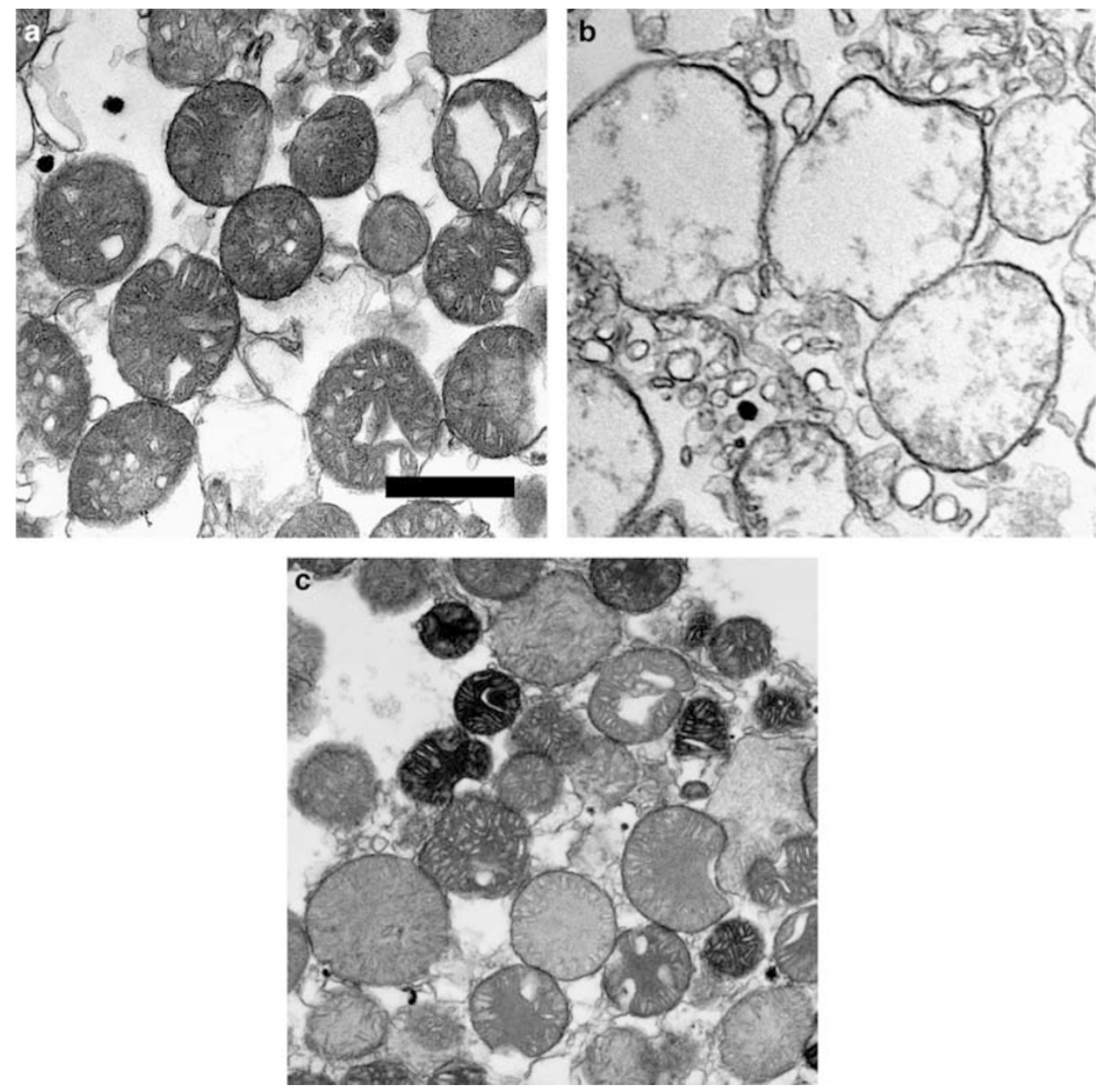

Figure 6 Electron microscope images of isolated mitochondria prepared freshly or frozen in mannitol/sucrose or trehalose. The mitochondria in the fresh and trehalose samples (a and $\mathbf{c}$ ) were nearly all in the condensed state with outer and inner membranes close together. The mannitol/sucrose mitochondria (b) show large-amplitude swelling and degraded cristae; however, outer membranes did not display obvious breaks. The bar represents $1 \mu \mathrm{M}$

Hackenbrock ${ }^{16}$; examples in Kuwana et al., ${ }^{10}$ and von Ahsen et $a{ }^{17}{ }^{17}$ ) In condensed forms, the matrix is more electron dense, indicative of more tightly packed material. Concomitantly, the intracristal space is more expanded and in contrast to the matrix is quite light. In this condensed state, the IMS, the region bounded by the mitochondrial outer and inner boundary membranes, is often (but not always) expanded, that is, the inner boundary membrane is pulled away from the outer membrane. The freshly prepared and trehalose-frozen mitochondria shown in Figure 6(a and c) are nearly all in the condensed state, with the freshly prepared mitochondria appearing slightly more condensed (generally darker matrix and more expanded intracristal spaces). In both samples, the outer membrane is seen to be in close apposition to the inner boundary membrane. In contrast, the mitochondria frozen in mannitol/sucrose exhibited significant structural damage. Most of the mitochondria were highly swollen (example of this type of swelling in von Ahsen et $a{ }^{17}{ }^{17}$ ) and contained degraded cristae (Figure 6b). Even with this large-amplitude swelling, often no breaks in outer membranes could be seen. In summary, no significant differences in ultrastructure were apparent between the freshly prepared and trehalose samples, whereas the mannitol/sucrose mitochondria were extensively damaged.

Bioenergetic function is partially preserved in trehalosefrozen mitochondria. To assess quantitatively the functional integrity of the inner membrane, we measured respiration of mitochondria in different metabolic states (Figure 7). Trehalose-frozen mitochondria showed significantly decreased rates of both phosphorylating (state 3) and maximally uncoupled respiration (Figure 7a) that resulted in decreased respiratory control and acceptor control ratios (Figure 7b). However, these mitochondria displayed essentially intact activity of cytochrome $c$ oxidase (Figure 7a). Taken together these data indicate that freezing in trehalose partially preserves bioenergetic function, consistent with the ability of these mitochondria to maintain a membrane potential (Figure 4b) and, therefore, to perform membrane potential-dependent functions: ATP synthesis (Figure 3) and protein import (Figure 5), as well as $\mathrm{Ca}^{2+}$ accumulation with consequent opening of the PTP (Figure 4a). 
a
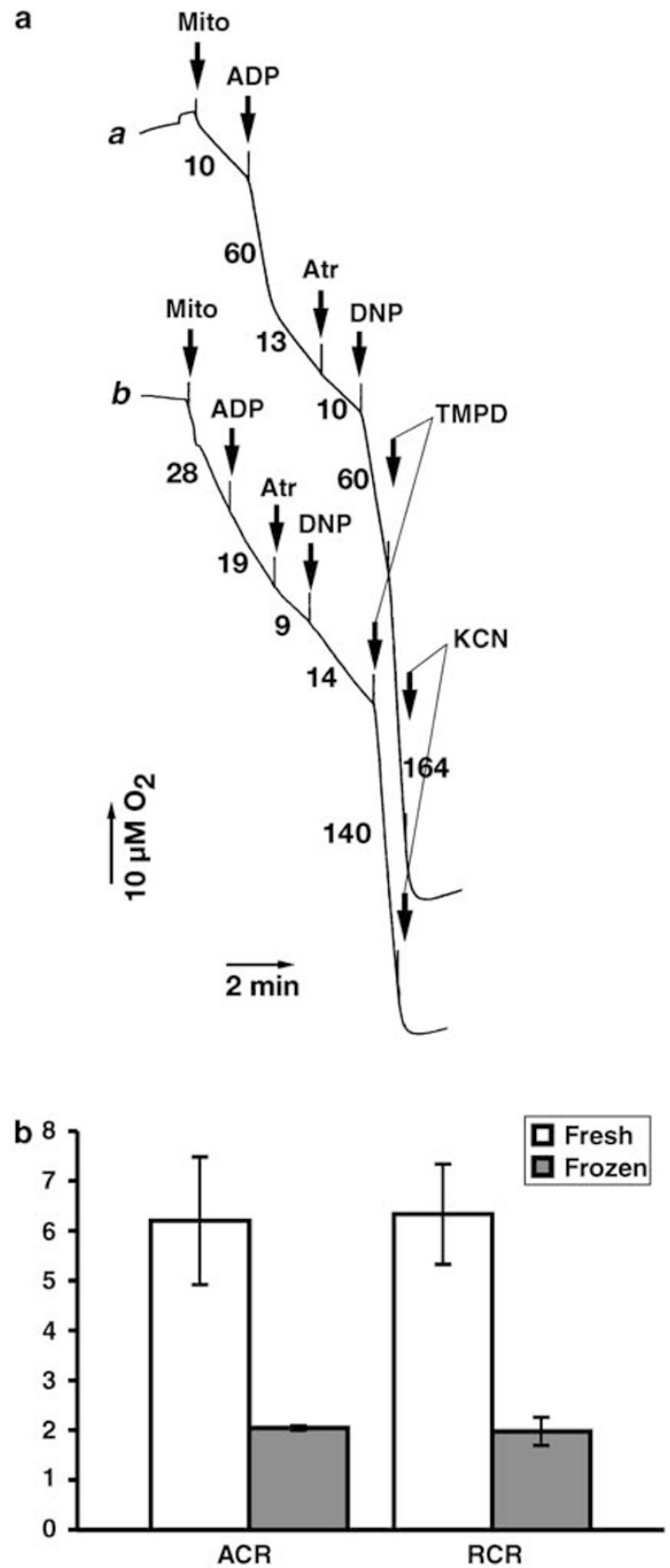

Figure 7 Bioenergetic function is compromised but not completely disrupted in trehalose-frozen mitochondria. (a) Respiration of freshly prepared and trehalosefrozen mitochondria. $1 \mathrm{mg} / \mathrm{ml}$ of freshly prepared (trace a) or trehalose-frozen (trace b) mouse liver mitochondria were incubated as described in Material and Methods. ADP $(200 \mu \mathrm{M} ; A D P)$ was used to induce metabolic state 3 (phosphorylating respiration). Next, $100 \mu \mathrm{M}$ atractyloside (Atr), an inhibitor of the adenine nucleotide translocator, was added to induce resting (state 4) respiration, and then $40 \mu \mathrm{M}$ DNP was added to measure the maximal rates of electron transport chain activity (state $3 \mathrm{u}$ or uncoupled respiration). Additions of $2 \mathrm{mM} \mathrm{N}, N, N^{\prime}, N^{\prime}$-tetramethyl-1,4phenylenediamine (TMPD) and $0.5 \mathrm{mM}$ potassium cyanide $(\mathrm{KCN})$ were used to assess cytochrome oxidase activity and nonmitochondrial oxygen consumption, respectively. The data are a paired experiment representative of three separate preparations. (b) Respiratory control in freshly prepared and trehalose-frozen mitochondria. Acceptor control ratios (ACR) and respiratory control ratios (RCR) were calculated as the ratios of rates of respiration in metabolic state 3 or state $3 u$, respectively, to the rates of respiration in metabolic state 4 . The data are mean \pm S.E.M., $n=3$

\section{Discussion}

We have shown that mouse liver mitochondria can be frozen in trehalose buffer in such a way that much of their biological function and ultrastructure is preserved. In particular, this procedure maintains the integrity of the MOM and the regulation of its permeability by $\mathrm{Bcl}-2$-family proteins. In contrast, outer membranes of mitochondria frozen in standard sucrose-mannitol buffer deteriorate quickly when thawed and then incubated at $37^{\circ} \mathrm{C}$. In one experiment, nearly $97 \%$ of cytochrome $c$ was lost from mitochondria during the first $15 \mathrm{~min}$ of incubation at $37^{\circ} \mathrm{C}$. Interestingly, the same mitochondria incubated at $22{ }^{\circ} \mathrm{C}$ retained over $80 \%$ of cytochrome $c$ for $45 \mathrm{~min}$. One possible reason for this temperature dependence of cytochrome $c$ release may be that mitochondria frozen in sucrose-mannitol buffer already have compromised MOMs and are simply more unstable during the $37^{\circ} \mathrm{C}$ incubation, perhaps as a result of increased membrane fluidity. However, a more intriguing possibility is that a temperature-dependent change in the structure of mitochondrial cristae occurs, similar to the changes observed by Korsmeyer and colleagues. ${ }^{9}$

Temperature also caused differences in the mitochondrial response to N/C-Bid; at $37^{\circ} \mathrm{C}, 10 \mathrm{nM} \mathrm{N} / \mathrm{C}$-Bid induced the complete release of cytochrome $c$ and Omi from the IMS in $24 \mathrm{~min}$, whereas at $22^{\circ} \mathrm{C}$, mitochondria released neither cytochrome $c$ nor Omi over 45 min (Figure 2). Furthermore, Bak oligomerization was observed only at $37^{\circ} \mathrm{C}$ and not at $22^{\circ} \mathrm{C}$ (data not shown). One possible explanation might be that the fluidity of the MOM is lower at $22^{\circ} \mathrm{C}$, thus hindering Bak-mediated lipidic pore formation. Alternatively, some unknown enzymatic activity might be required.

Interestingly, mitochondria from Xenopus laevis eggs are inherently resistant to freeze-thaw even in mannitol-sucrose buffers, retaining most of their cytochrome $c$ content and their ability to be permeabilized by proapoptotic Bcl-2-family proteins. ${ }^{15,18}$ It is possible that Xenopus eggs, which must be fertilized outside the mother's body, contain protectants like trehalose to help them resist adverse conditions in the wild, such as freezing or desiccation.

It also seems likely that temperature played a role in lowering the ATP concentration of trehalose-frozen mitochondria during storage (Figure 3). As mitochondrial ATP synthesis is a temperature-dependent reaction requiring the activities of proton pumps, whereas ATP hydrolysis can take place even at low temperatures, freezing could potentially tip the balance towards net loss of ATP. Despite their lowered initial ATP concentrations, however, when trehalose-frozen mitochondria were stimulated with ADP or ADP + G3P, they reached final ATP concentrations comparable to those seen with fresh mitochondria within $30 \mathrm{~min}$ (Figure 3). Thus, our data indicate that trehalose-frozen mitochondria are capable of ATP synthesis and can maintain normal ATP levels when warmed to $37^{\circ} \mathrm{C}$.

Closer examination of mitochondrial respiration revealed that the inner membrane function of trehalose-frozen mitochondria is somewhat compromised (Figure 7) as reflected by their low respiratory control ratios. Freezing the mitochondria appeared primarily to compromise rates of phosphorylating (ADP-stimulated) and uncoupler-stimulated 
(DNP-stimulated) respiration rather than dramatically increasing the extent of mitochondrial uncoupling (measured in the presence of atractyloside). The reason for this is unclear; however, one possibility is that freezing disrupts the interaction of the proteins that form one or more of the electron transport chain complexes, yet has a minimal effect on the integrity of the lipid membrane. As well, frozen mitochondria may release some of their matrix solutes, such as ADP and NAD/NADH. A high extramitochondrial concentration of ADP could explain, for example, the finding that the mitochondria were in state 3 from the start (i.e., they displayed a high initial rate of oxygen consumption that was not increased by exogenous ADP.) Nevertheless, trehalose-frozen mitochondria can maintain sufficient transmembrane potential to synthesize ATP (Figure 3), to import and process mitochondrial precursor proteins (Figure 5) and to swell in response to elevated external $\mathrm{Ca}^{2+}$ (Figure 4), indicating a substantial degree of functional preservation. Furthermore, electron microscopy showed that the ultrastructure of trehalose-frozen mitochondria was largely intact (Figure 6).

While this manuscript was in preparation, a publication appeared that described the loading of liver mitochondrial matrix with trehalose via the intentional reversible opening of the PTP using $150 \mu \mathrm{M}$ calcium, a manipulation that was reported to facilitate dessication of mitochondria. ${ }^{19}$ The claim was made that trehalose-loaded and desiccated mitochondria retain a high degree of inner membrane integrity. However, no direct comparison with freshly isolated mitochondria was made, nor was the integrity of the MOM examined. Indeed, the activation of permeability transition, at least under some conditions, is known to rupture the MOM. ${ }^{15}$ Thus, it is possible that mitochondria that are loaded with trehalose via induction of PT may better preserve inner membrane function, but perhaps at the expense of compromising outer membrane integrity and possibly the content of other solutes in the matrix that could, in principle, leak out through the PTP.

Our method of freezing isolated mitochondria in the presence of trehalose preserves a number of their important biological activities, in particular the regulation of $\mathrm{MOM}$ permeability by Bcl-2-family proteins. The protocol developed here will make the use of isolated mitochondria more convenient for research on apoptosis and other mitochondrial functions.

\begin{abstract}
Materials and Methods
Isolation and storage of mouse liver mitochondria. Mouse liver mitochondria were isolated by Dounce homogenization in AT buffer $(300 \mathrm{mM}$ trehalose, $10 \mathrm{mM}$ HEPES-KOH pH 7.7, $10 \mathrm{mM} \mathrm{KCl}, 1 \mathrm{mM}$ EGTA, $1 \mathrm{mM}$ EDTA and $0.1 \% \mathrm{BSA})$. The homogenate was centrifuged at $600 \times \mathrm{g}$ for $10 \mathrm{~min}$. The supernatant was removed and centrifuged at $3500 \times g$ for $15 \mathrm{~min}$, and the resulting pellet was resuspended in $10 \mathrm{ml}$ of isolation buffer and centrifuged at $1500 \times \mathrm{g}$ for $5 \mathrm{~min}$. The mitochondrial supernatant was centrifuged at $5500 \times g$ for $10 \mathrm{~min}$, and the last two steps were repeated. The mitochondrial pellet was resuspended in a small volume of trehalose buffer at $50 \mathrm{mg}$ of protein $/ \mathrm{ml}$. For freezing, $5-\mu \mathrm{l}$ samples of mitochondrial suspension were pipetted into 0.6-ml Eppendorf tubes placed on the bed of dry ice. It was important to avoid touching the tube wall with the pipet tips, as this could cause mitochondria inside the tip to freeze immediately. The tubes were immediately thrown into liquid nitrogen and then stored at $-80^{\circ} \mathrm{C}$. For defrosting, tubes were held between the fingers for rapid thawing.
\end{abstract}

Cytochrome $c$ release assay. Frozen or freshly isolated mitochondrial pellets were washed once in AT buffer containing $80 \mathrm{mM} \mathrm{KCl}$ and resuspended in the same buffer supplemented with an energy regenerating system $(5 \mathrm{mM}$ succinate, $2 \mathrm{mM}$ ATP, $10 \mu \mathrm{M}$ phosphocreatine and $10 \mu \mathrm{g} / \mathrm{ml}$ creatine kinase). Samples were incubated with the indicated amounts of recombinant proteins or peptides at $37^{\circ} \mathrm{C}$ for $30 \mathrm{~min}$ unless otherwise specified. For the cytochrome $c$ release assay, we typically used $30-60 \mu \mathrm{g}$ of mitochondria in $30 \mu \mathrm{l}$ of trehalose buffer. Mitochondrial pellets were separated from supernatants by centrifugation at $10000 \times g$ for $10 \mathrm{~min}$ at $4^{\circ} \mathrm{C}$. Pellets and supernatants were suspended in SDS sample buffer and separated by $15 \%$ sodium dodecyl sulfate-polyacrylamide gel electrophoresis (SDS-PAGE), then immunoblotted onto PVDF membrane using anti-cytochrome $c$ antibody (BD Bioscience). In some experiments, the membranes were reprobed with antibodies to Htra2/Omi (R\&D Systems) or VDAC (BD Bioscience).

ATP bioluminescence assay. Mitochondrial ATP concentrations were measured as described. ${ }^{20}$ Fresh or frozen mitochondria pellets were resuspended in $75 \mu \mathrm{l}$ of ATP synthesis buffer $\left(20 \mathrm{mM}\right.$ Tris- $\mathrm{HCl} \mathrm{pH} 7.4,15 \mathrm{mM} \mathrm{KH}_{2} \mathrm{PO}_{4}, 0.6 \mathrm{M}$ sorbitol, $10 \mathrm{mM} \mathrm{MgSO}_{4}$ and $2.5 \mathrm{mg} / \mathrm{ml} \mathrm{BSA}$ ). To induce ATP synthesis, $67 \mu \mathrm{M} \mathrm{ADP}$ (with or without G3P) was added. Where indicated, mitochondrial ATP synthesis was inhibited by the addition of antimycin $\mathrm{A}(0.2 \mu \mathrm{M})$. After incubation at $37^{\circ} \mathrm{C}$ for $30 \mathrm{~min}, 1.75 \mu \mathrm{l}$ of $60 \%$ perchloric acid was added, and samples were left on ice for $10 \mathrm{~min}$ followed by centrifugation at $15000 \times \mathrm{g}$ for $10 \mathrm{~min}$. A volume of $60 \mu \mathrm{l}$ was collected and neutralized with $11.5 \mu \mathrm{l}$ of $1 \mathrm{M} \mathrm{KOH}$. The reaction was incubated on ice for $5 \mathrm{~min}$ and centrifuged as before. Finally, the ATP concentration for $10 \mu \mathrm{l}$ of the resulting supernatant was determined using the ATP Bioluminescence Assay Kit CLS II (Roche) according to the manufacturer's instructions.

Calcium-induced mitochondrial swelling. Mitochondrial pellets were suspended (at $500 \mu \mathrm{g} / \mathrm{ml}$ as protein) in assay buffer containing $150 \mathrm{mM} \mathrm{KCl}, 5 \mathrm{mM}$ $\mathrm{KH}_{2} \mathrm{PO}_{4}$ and $5 \mathrm{mM}$ Tris- $\mathrm{HCl}$ (pH 7.4). A pair of samples, one containing $25 \mu \mathrm{m}$ cyclosporin $\mathrm{A}$ and the other without, were placed in cuvettes side by side in a Cary 100 Bio UV-Visible Spectrophotometer and light scattering measurements at $540 \mathrm{~nm}$ were taken from both samples simultaneously. Measurements were taken five times every second. Two minutes after the initiation of the assay, $100 \mu \mathrm{M}$ of $\mathrm{CaCl}_{2}$ ( $\sim 200 \mathrm{nmol} / \mathrm{mg}$ of mitochondria) was added to both samples and the assay was continued for another $8 \mathrm{~min}$.

Flow cytometric analysis of isolated liver mitochondria. Mitochondrial transmembrane potential was measured as described. ${ }^{21}$ Fresh or frozen mitochondrial pellets were suspended in phosphate-buffered saline (PBS). TMRE (100 nM) was added to the suspension, which was mixed well and was immediately analyzed by flow cytometry, with care taken to gate out noise from particulate contaminants. Addition of $1-10 \mu \mathrm{M} \mathrm{CCCP} \mathrm{resulted} \mathrm{in} \mathrm{an} \mathrm{immediate}$ downward shift in TMRE staining.

Mitochondrial protein import assay. Frozen mitochondrial pellets were treated as in the cytochrome $c$ release assay. Twenty-microliter samples containing $0.2 \mu \mathrm{l}$ of the indicated ${ }^{35} \mathrm{~S}$ methionine-labeled proteins, prepared in rabbit reticulocytes, were incubated at $37^{\circ} \mathrm{C}$ for the indicated time. Pellets were precipitated as before and supernatants and pellets were subjected to $12 \%$ SDSPAGE. Gels were dried and exposed overnight with Kodak MR film.

Electron microscopy. Electron microscopy was performed essentially as described. ${ }^{10,17,22}$ Briefly, fresh or frozen resuspended mitochondrial pellets $(1.5 \mathrm{mg} /$ $\mathrm{ml}$ protein) isolated by differential centrifugation (thus containing contaminating ER and Golgi membranes) were incubated in $1 \%$ formaldehyde on ice for $10 \mathrm{~min}$, and centrifuged at $6000 \times g$ for $5 \mathrm{~min}$. Pellets were fixed with $2 \%$ paraformaldehyde$2.5 \%$ glutaraldehyde in $0.15 \mathrm{M}$ sodium cacodylate buffer, $\mathrm{pH} 7.4$ for $1 \mathrm{~h}$ on ice. Afterwards, they were rinsed in ice-cold $0.15 \mathrm{M}$ sodium cacodylate buffer containing $3 \mathrm{mM}$ calcium chloride for membrane preservation. Postfixation was accomplished with ice-cold $1 \%$ osmium tetroxide in $0.15 \mathrm{M}$ sodium cacodylate containing $0.8 \%$ potassium ferrocyanide and $3 \mathrm{mM}$ calcium chloride. Following repeated rinses in ice-cold, double-distilled water, the mitochondria were stained and internal membranes were stabilized in ice-cold $2 \%$ uranyl acetate. Dehydration was initiated with a graded ethanol series on ice $-20,50,70$ and $90 \%$. Dehydration was completed at room temperature with $100 \%$ ethanol. Infiltration was performed with well-mixed $50 \%$ ethanol/50\% Durcupan ACM resin. Infiltration continued with multiple exchanges of $100 \%$ Durcupan ACM. The resin was hardened in a $60^{\circ} \mathrm{C}$ oven. Sections from the samples were cut with a thickness of $\sim 80 \mathrm{~nm}$ using a Leica ultramicrotome. After poststaining in uranyl acetate and lead salts, these sections 
were examined using a JEOL 1200FX electron microscope. Images were recorded on film at $6000 \times$ magnification. The negatives were scanned at $4000 \mathrm{dpi}$ using a Nikon CoolScan giving a pixel size of $0.96 \mathrm{~nm}$. Contrast adjustment was performed with Adobe Photoshop.

Measurement of respiration. Respiration of mitochondria was measured using a Clark-type oxygen electrode (Diamond General) connected to an oxygen monitor (Yellow Springs Instruments) in incubation medium containing $250 \mathrm{mM}$ sucrose, $2 \mathrm{mM}$ inorganic phosphate, $1 \mathrm{mM}$ EGTA, $2 \mathrm{mM} \mathrm{MgCl}_{2}$ and $10 \mathrm{mM}$ HEPES- $\mathrm{KOH}, \mathrm{pH} 7.4$ at $22^{\circ} \mathrm{C}$. Glutamate $(5 \mathrm{mM})$ and malate $(5 \mathrm{mM})$ were used as respiratory substrates.

Acknowledgements. We thank Jean-Ehrland Ricci for his help in flow analysis of isolated mitochondria, Mary Chamberlin of Ohio University for helpful discussions and Lisa Bouchier-Hayes for critical reading of the manuscript. This work was supported by NIH Grants GM50284 and GM62289 to DDN. Some of the work included here was conducted at the National Center for Microscopy and Imaging Research, which is supported by NIH Grant RR04050 to MHE. AA was supported by LIPID MAPS Large scale Collaborative Grant GM069338 from NIH.

1. Nukala VN, Singh IN, Davis LM, Sullivan PG. Cryopreservation of brain mitochondria: a novel methodology for functional studies. J Neurosci Methods 2006; 152: 48-54.

2. Kuwana T, Newmeyer DD. Bcl-2-family proteins and the role of mitochondria in apoptosis. Curr Opin Cell Biol 2003; 15: 691-699.

3. Newmeyer DD, Ferguson-Miller S. Mitochondria. Releasing power for life and unleashing the machineries of death. Cell 2003; 112: 481-490.

4. Green DR. Apoptotic pathways: ten minutes to dead. Cell 2005; 121: 671-674.

5. Henry-Mowatt J, Dive C, Martinou JC, James D. Role of mitochondrial membrane permeabilization in apoptosis and cancer. Oncogene 2004; 23: 2850-2860.

6. Elbein AD, Pan YT, Pastuszak I, Carroll D. New insights on trehalose: a multifunctiona molecule. Glycobiology 2003; 13: 17R-27R.

7. Crowe LM, Mouradian R, Crowe JH, Jackson SA, Womersley C. Effects of carbohydrates on membrane stability at low water activities. Biochim Biophys Acta 1984; 769: 141-150.
8. Rudolph AS, Crowe JH. Membrane stabilization during freezing: the role of two natural cryoprotectants, trehalose and proline. Cryobiology 1985; 22: 367-377.

9. Scorrano L, Ashiya M, Buttle K, Weiler S, Oakes SA, Mannella CA et al. A distinct pathway remodels mitochondrial cristae and mobilizes cytochrome $c$ during apoptosis. Dev Cell 2002; 2: 55-67.

10. Kuwana T, Mackey MR, Perkins G, Ellisman MH, Latterich M, Schneiter R et al. Bid, bax and lipids cooperate to form supramolecular openings in the outer mitochondria membrane. Cell 2002; 111: 331-342.

11. Kuwana T, Bouchier-Hayes L, Chipuk JE, Bonzon C, Sullivan BA, Green DR et al. BH3 domains of $\mathrm{BH} 3-$ only proteins differentially regulate Bax-mediated mitochondria membrane permeabilization both directly and indirectly. Mol Cell 2005; 17: 525-535.

12. Szabadkai G, Rizzuto R. Participation of endoplasmic reticulum and mitochondrial calcium handling in apoptosis: more than just neighborhood? FEBS Lett 2004; 567: 111-115.

13. Hajnoczky G, Davies E, Madesh M. Calcium signaling and apoptosis. Biochem Biophys Res Commun 2003; 304: 445-454.

14. Rizzuto R, Pinton P, Ferrari D, Chami M, Szabadkai G, Magalhaes PJ et al. Calcium and apoptosis: facts and hypotheses. Oncogene 2003; 22: 8619-8627.

15. von Ahsen O, Newmeyer DD. Cell-free apoptosis in Xenopus laevis egg extracts. Methods Enzymol 2000; 322: 183-198.

16. Hackenbrock $\mathrm{CR}$. Chemical and physical fixation of isolated mitochondria in low-energy and high-energy states. Proc Natl Acad Sci USA 1968; 61: 598-605

17. von Ahsen O, Renken C, Perkins G, Kluck RM, Bossy-Wetzel E, Newmeyer DD. Preservation of mitochondrial structure and function after Bid- or Bax-mediated cytochrome c release. J Cell Biol 2000; 150: 1027-1036.

18. Kluck RM, Bossy-Wetzel E, Green DR, Newmeyer DD. The release of cytochrome $c$ from mitochondria: a primary site for Bcl-2 regulation of apoptosis. Science 1997; 275: 1132 1136

19. Liu XH, Aksan A, Menze MA, Hand SC, Toner M. Trehalose loading through the mitochondrial permeability transition pore enhances desiccation tolerance in rat liver mitochondria. Biochim Biophys Acta 2005; 1717: 21-26.

20. Allemann N, Schneider A. ATP production in isolated mitochondria of procyclic Trypanosoma brucei. Mol Biochem Parasitol 2000; 111: 87-94.

21. Mattiasson G. Flow cytometric analysis of isolated liver mitochondria to detect changes relevant to cell death. Cytometry $A$ 2004; 60A: 145-154

22. Kluck RM, Esposti MD, Perkins G, Renken C, Kuwana T, Bossy-Wetzel E et al. The pro-apoptotic proteins, Bid and Bax, cause a limited permeabilization of the mitochondrial outer membrane that is enhanced by cytosol. J Cell Biol 1999; 147: 809-822. 